$13^{\text {th }}$ International Conference on

\title{
Subband Coding of Speech Signals Using Decimation and Interpolation
}

\begin{abstract}
Ashraf M. Aziz ${ }^{*}$
Abstract: In many practical applications of digital signal processing, such as telecommunication systems, one is faced with the problem of changing the sampling rate of a signal, either increasing it or decreasing it by some amount. In telecommunication systems, that transmit and receive different types of signals, there is a requirement to process the various signals at different rates commensurate with the corresponding bandwidths of the various signals. This paper addresses the problem of sampling rate conversion and multirate signal processing in the digital domain. A structure of a two-channel quadrature mirror filter with low pass filter, high pass filter, decimators and interpolators, is proposed to perform subband coding of speech signals in the digital domain. The proposed structure decomposes a signal into low frequency and high frequency components. The performance of the proposed structure is compared with the performance of the delta-modulation encoding systems. The results show that the proposed structure significantly reduces the error and achieves considerable performance improvement compared to delta-modulation encoding systems.
\end{abstract}

Keywords: Sampling rate conversion; Multirate digital signal processing ; Data compression.

\section{Introduction}

In telecommunication systems that transmit and receive different types of signals; e.g., teletype, facsimile, speech, video, etc., there is a requirement to process the various signals at different rates. The process of converting a signal from a given rate to a different rate is called sampling rate conversion. In turn, systems that employ multiple sampling rates in digital signal processing are called multirate digital signal processing (DSP) systems [1, 2].

Sampling rate conversion of a digital signal can be accomplished in one of two methods. The first method is to convert the digital signal into an analog signal and then resample the analog signal at a different rate. In this case the digital signal is passed through a digital-to-analog (D/A) converter, filter it if necessary. Then the output analog signal from the D/A converter is resampled at the desired rate, i.e., the resulting analog signal is passed through an analog-todigital (A/D) converter. The advantage of this method is that the new sampling rate can be arbitrarily selected and need not have any special relationship to the old sampling rate.

The major disadvantage of this method is the signal distortion introduced by the D/A converter in the signal reconstruction, and by the quantization effects in the A/D conversion. The second method of sampling rate conversion performs the sampling rate conversion entirely in the digital domain. This method avoids the major disadvantages of the first method $[3,4]$.

\footnotetext{
* Egyptian Armed Forces
} 
Figure 1 describes the process of sampling rate conversion in the digital domain as a linear filtering process. The input signal $x(n)$ is characterized by the sampling rate $F_{x}=1 / T_{x}$, where $T_{x}$ is the sampling interval of the input signal. The output signal $y(n)$ is characterized by the sampling rate $F_{y}=1 / T_{y}$. The ratio $F_{y} / F_{x}=I / D$ is considered to be rational, where $D$ and $I$ are relatively prime integers. The sampling rate conversion process can also be understood from the point of view of digital resampling of an input analog signal $x(t)$. If we assume that the analog signal $x(t)$ is sampled at a rate $F_{x}$ to generate $x(n)$, the goal of sampling rate conversion is to obtain another sequence $y(m)$ at another rate $F_{y}$. It is clear that the sequence $y(m)$ is a time-shifted version of $x(n)$. Such a time shift can be realized by using a linear filter that has a flat magnitude response and a linear phase response. If the two sampling rates are not equal, the required amount of time shifting will vary from sample to sample. Thus the rate converter can be implemented using a set of linear filters that have the same flat magnitude response but generate different time delays.

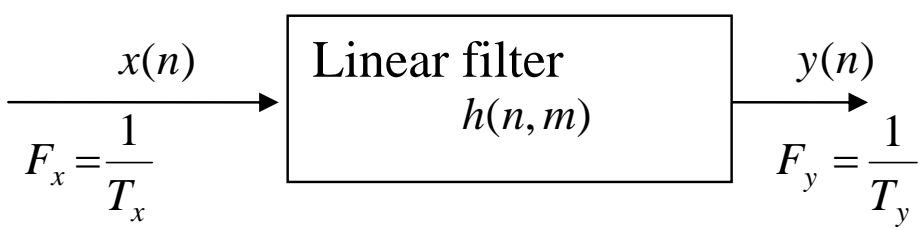

Fig. 1 Sampling rate conversion.

The case of sampling rate reduction by an integer factor $D$ (downsampling by $D$ ) is called decimation. The process of increasing the sampling rate by an integer $I$ (upsampling by $I$ ) is called interpolation. The general case of sampling rate conversion is to convert the input sequence by a rational factor $\frac{I}{D}[1,5]$.

In this paper we address the problem of decomposing a signal into low frequency and high frequency components and its use in data compression by performing decimation and interpolation in the frequency domain. The remainder of this paper is organized as follows. A brief review of decimation and interpolation of a digital signal is addressed in Section 2. In Section 3, a structure using decimators, interpolators, low and high pass filters, is presented to perform data compression. In section 4, The performance of the structure is evaluated and compared with the delta modulation data compression systems. Finally, Section 5 contains conclusions.

\section{Multirate DSP Using Decimation and Interpolation}

\subsection{Decimation}

Decimation of a signal $x(n)$ by a factor $D$ means that its sampling rate is reduced by a factor $D$. This process is called downsampling. Let us assume that the signal $x(n)$ with spectrum $X(\omega)$ is to be down sampled by an integer factor $D$. The spectrum $X(\omega)$ is assumed to be nonzero in the frequency interval $0 \leq|\omega| \leq \pi$ or equivalently, $|F| \leq \frac{F_{X}}{2}$. It is required to reduce the sampling rate simply by selecting every $D^{\text {th }}$ value of $x(n)$. The resulting signal 
will be an aliased version of $x(n)$, with a folding frequency of $\frac{F_{x}}{2 D}$. To avoid aliasing, the bandwidth of $x(n)$ must be reduced to $F_{\max }=\frac{F_{x}}{2 D}$ or equivalently, to $\omega_{\max }=\frac{\pi}{D}$. In this case, the signal $x(n)$ is downsampled correctly and thus avoid aliasing. The decimation process is shown in Fig. 2 [1-3, 6] .

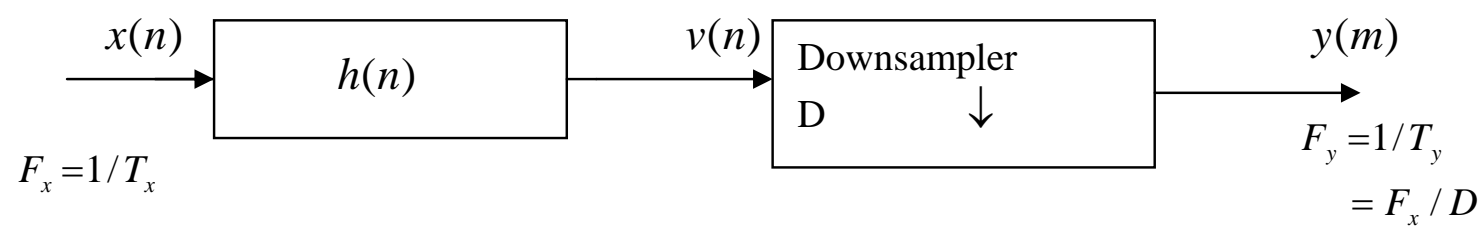

Fig. 2 Decimation by a factor of $D$.

The input sequence $x(n)$ is passed through a low pass filter to eliminate the spectrum of $X(\omega)$ in the range $\frac{\pi}{D} \leq \omega \leq \pi$. The implication is that only the spectrum of $x(n)$ in the range $\omega \leq \frac{\pi}{D}$ is of interest in further processing of the signal. The low pass filter is characterized by the impulse response $h(n)$ and a frequency response $H_{D}(\omega)$, given by

$H_{D}(\omega)= \begin{cases}1, & |\omega| \leq \pi / D \\ 0, & \text { otherwise }\end{cases}$

Using the $z$ - transform of the output sequence $y(m)$, it is easy to prove that [1-3]

$Y\left(\omega_{y}\right)=\frac{1}{D} \sum_{k=0}^{D-1} H_{D}\left(\frac{\omega_{y}-2 \pi k}{D}\right) X\left(\frac{\omega_{y}-2 \pi k}{D}\right)$.

The aliasing in (8) can be eliminated with a properly designed filter $H_{D}(\omega)$, consequently, all but the first term in (2) vanish. Hence

$Y(\omega)=\frac{1}{D} X\left(\frac{\omega}{D}\right), \quad 0 \leq|\omega| \leq \pi$

An example of an input sequence $x(n)$ and the output sequence after decimation, $y(m)$, assuming that $D=3$, is shown in Fig. 3. The corresponding spectra are shown in Fig. 4.

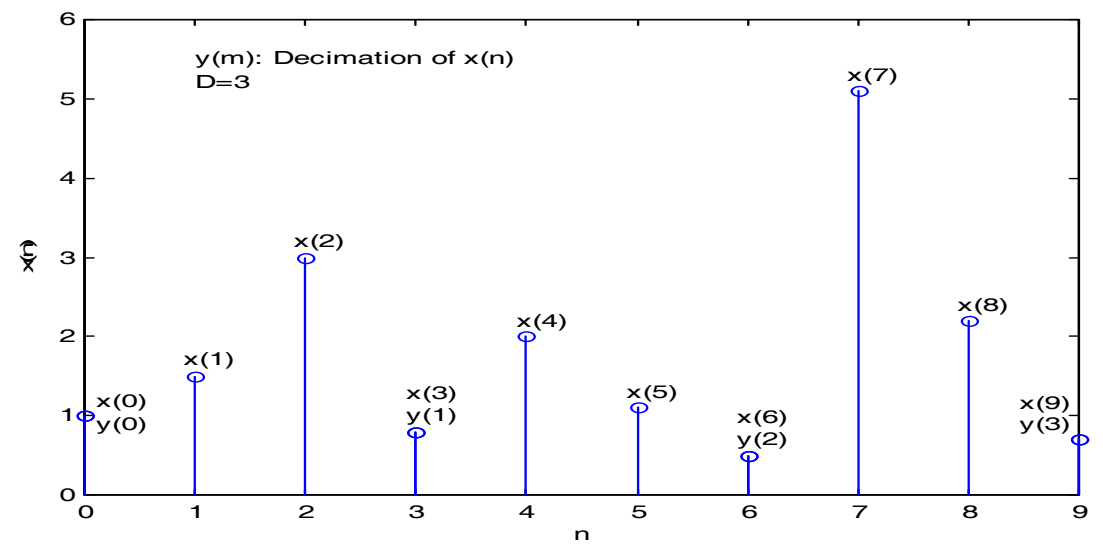

Fig. 3 Decimation of a sequence $x(n)$ by a factor of $D=3$. 


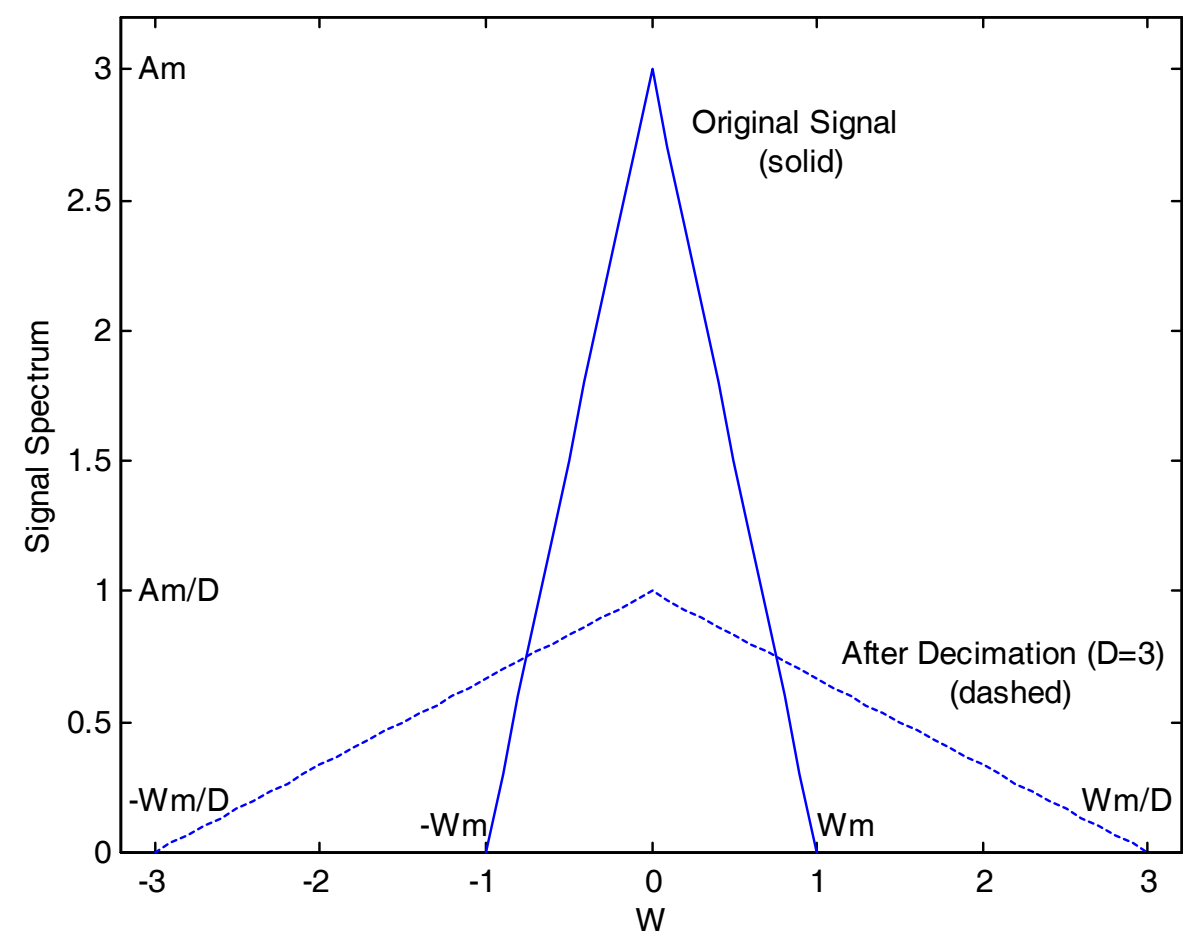

Fig. 4 Spectrum of the input sequence before and after decimation.

\subsection{Interpolation}

Interpolation of a signal $x(n)$ by an integer factor $I$ means that its sampling rate is increased by a factor $I$. The interpolation process is shown in Fig. $5[1-3,6]$. This process is called upsampling. Let us assume that the signal $x(n)$ with spectrum $X(\omega)$ is to be upsampled by an integer factor $I$. The spectrum $X(\omega)$ is assumed to be nonzero in the frequency interval $0 \leq|\omega| \leq \pi$. An increase in the sampling rate by an integer factor of $I$ can be accomplished by interpolating $I-1$ new samples between successive values of the signal. The interpolation process can be accomplished in a variety of ways. We consider the way that preserves the spectral shape of the signal sequence $x(n)[8,9]$.

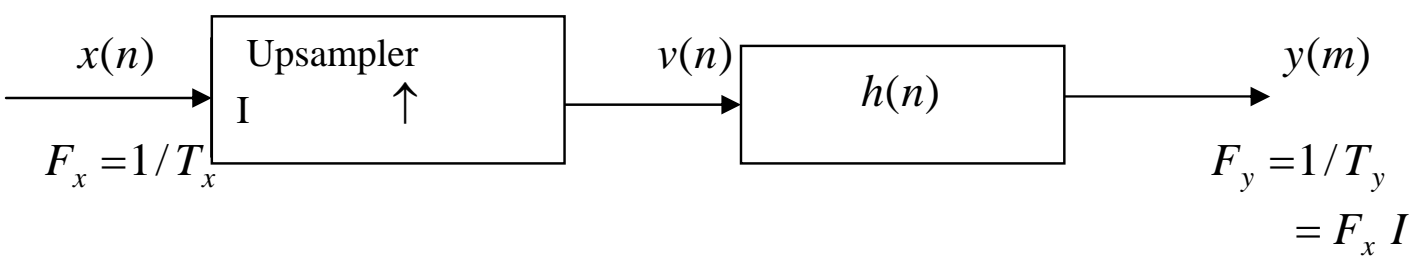

Fig. 5 Interpolation by a factor of $I$.

Let $v(m)$ denote a sequence with a rate $F_{y}=I F_{x}$, which is obtained from $x(n)$ by adding $I-1$ zeros between successive values of $x(n)$. Thus

$v(m)= \begin{cases}x(m / I), & m=0, \pm I, \pm 2 I, \ldots \ldots . \\ 0, & \text { otherwise }\end{cases}$ 
The sampling rate of $v(m)$ is identical to the rate of $y(m)$. Using the $z$ - transform of (10) we can deduce that [5]

$$
\begin{aligned}
& H_{I}(\omega)= \begin{cases}C, & 0 \leq|\omega| \leq \pi / I \\
0, & \text { otherwise }\end{cases} \\
& Y(\omega)=I X(\omega I), \quad 0 \leq|\omega| \leq \pi / I
\end{aligned}
$$

An example of an input sequence $x(n)$ and the output sequence after interpolation, $y(m)$, assuming that $I=3$, is shown in Fig. 6. The corresponding spectra are shown in Fig. 7.

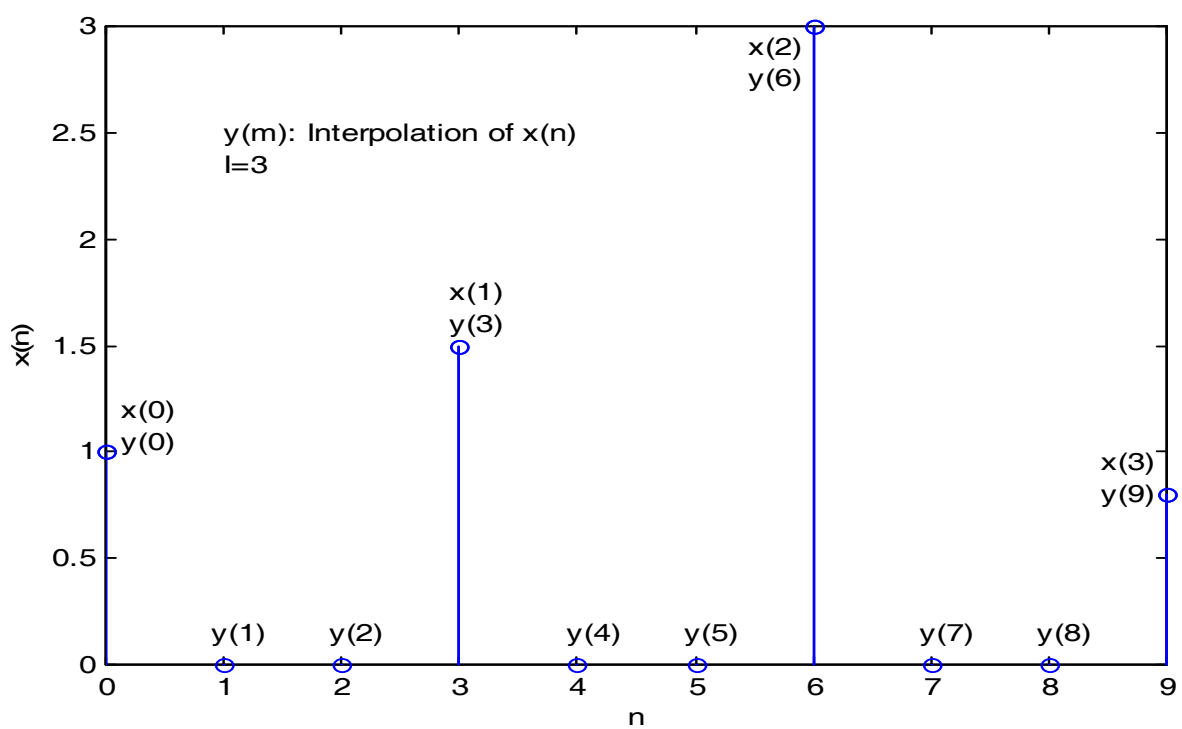

Fig. 6 Interpolation of a Sequence $x(n)$ by a factor of $I=3$.

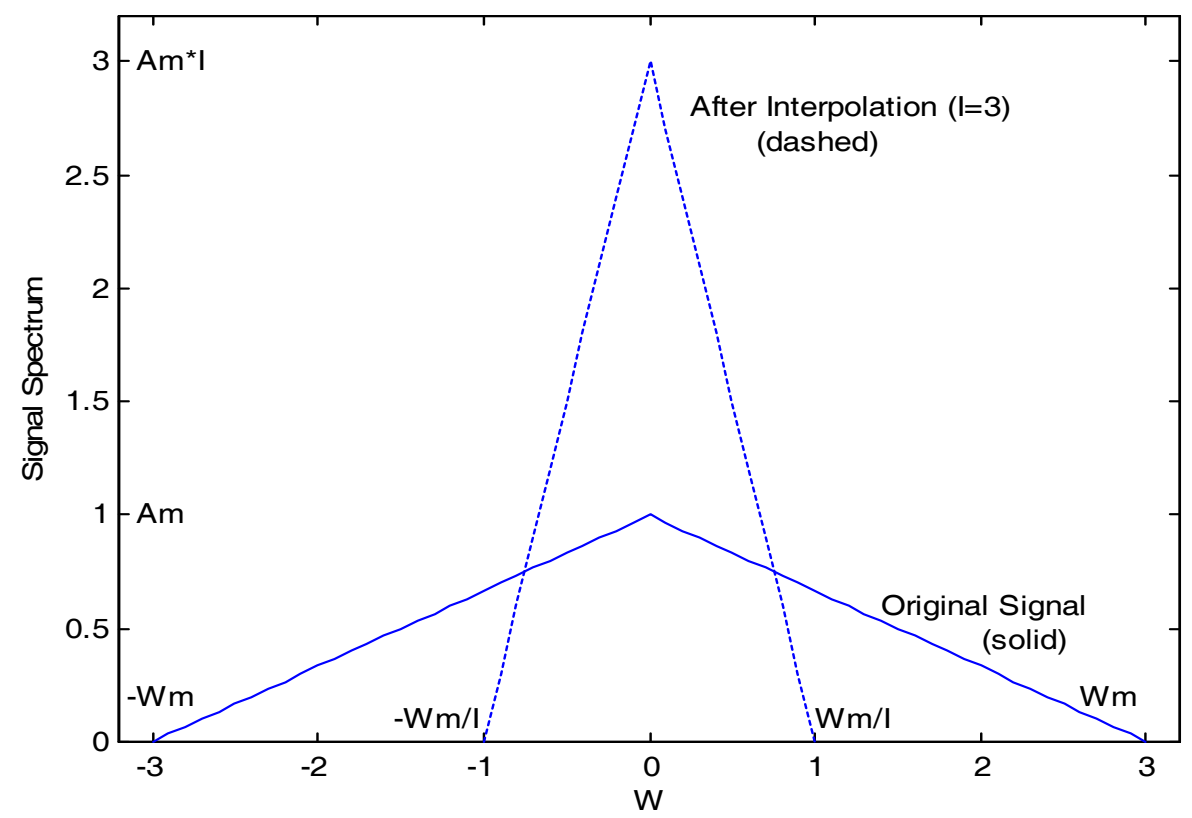

Fig. 7 Spectrum of the input sequence before and after interpolation. 


\section{Subband Coding Using Decimation and Interpolation}

Consider the structure of Figure 8. The speech signal is considered to be sampled at a rate $F_{s}$ samples per second. The first frequency subdivision splits the signal spectrum into two equal-width segments, a lowpass signal $\left(0 \leq F \leq F_{s} / 4\right)$ and a highpass signal $\left(F_{s} / 4 \leq F \leq F_{s} / 2\right)$. The second frequency subdivision splits the low pass signal from the first stage into two equal bands, a lowpass signal $\left(0 \leq F \leq F_{s} / 8\right)$ and a highpass signal $\left(F_{s} / 8 \leq F \leq F_{s} / 4\right)$. Finally, the third frequency subdivision splits the low pass signal from the second stage into two equal bandwidth signals. Thus the signal is subdivided into four frequency bands, covering three octaves, as shown in Fig. 8.

Decimation by a factor of 2 is performed after frequency division. By allocating a different number of bits per sample to the signal in the four subbands, we can achieve a reduction in the bit rate of the digitalized speech signal. For simplicity, we consider the case of one frequency subdivision as shown in Fig. 8. The filters designs are very important in achieving good performance in subband coding $[14,15]$. Aliasing resulting from decimation of the subband signals must be negligible. The two filters (lowpass and high pass filters) are called quadrature mirror filters (QMF) [16]. The decoding process for the subband encoded speech signal is basically the reverse of the encoding process. The signals in adjacent lowpass and highpass frequency bands are interpolated, filtered, and combined as shown in Fig. 9. A pair of QMF is used in the signal decoding section as shown in Fig. 8. Subband coding of signals is an effective method for achieving bandwidth compression in a digital representation of the signal, when the signal energy is encountered in a particular region of the frequency band. Multirate signal processing provides efficient implementation of the subband encoding.

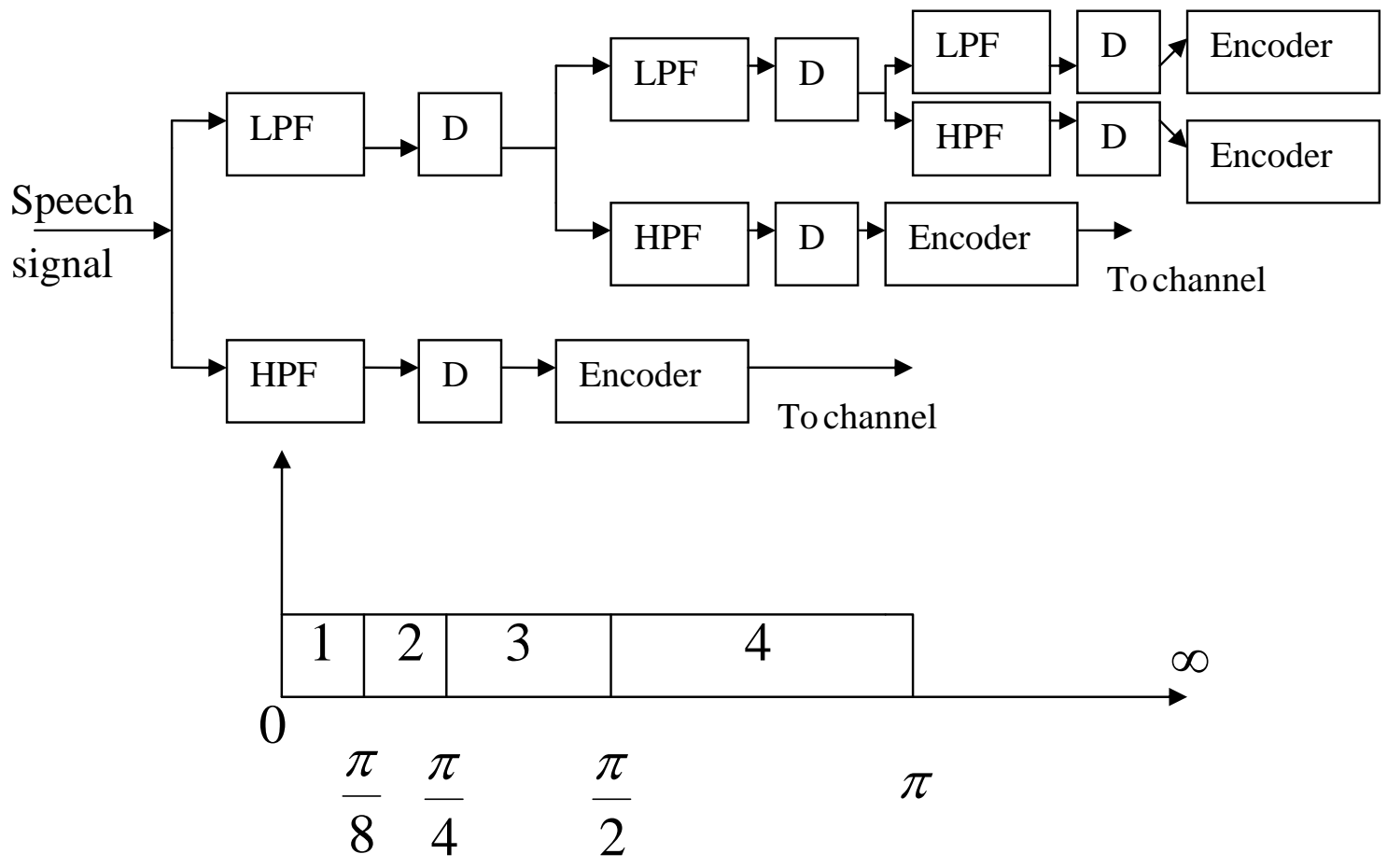

Fig. 8 block diagram of a subband speech encoder $(D=2)$ with three frequency subdivisions. 


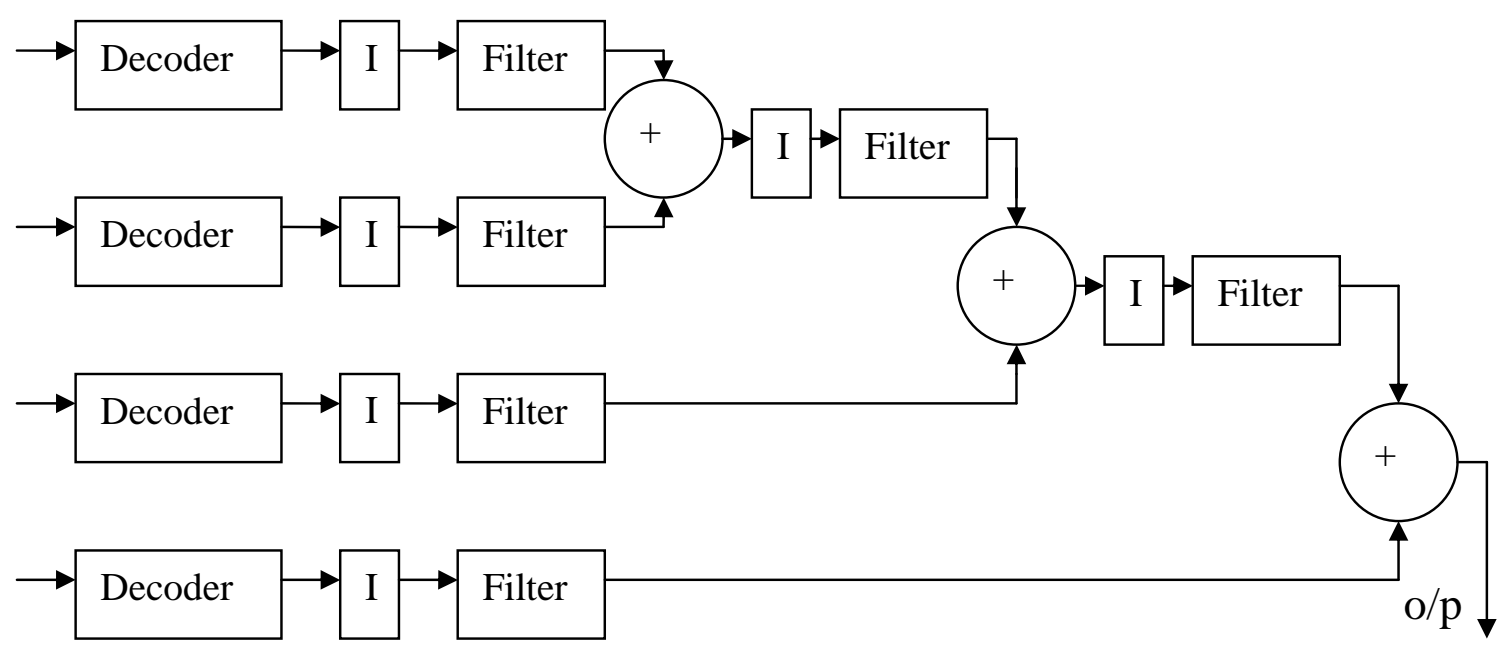

Fig. 9 Block diagram of a subband speech decoder $(I=2)$ with three frequency subdivisions.

The two-channel QMF shown in Fig. 10 is the basic building block in speech signal encoding. It employs two decimators in the signal encoding section and two interpolators in the signal decoding section. The lowpass and highpass filters in the encoding section have impulse responses $h_{0}(n)$ and $h_{1}(n)$, respectively. Similarly, the lowpass and highpass filters in the decoding section have impulse responses $g_{0}(n)$ and $g_{1}(n)$, respectively.

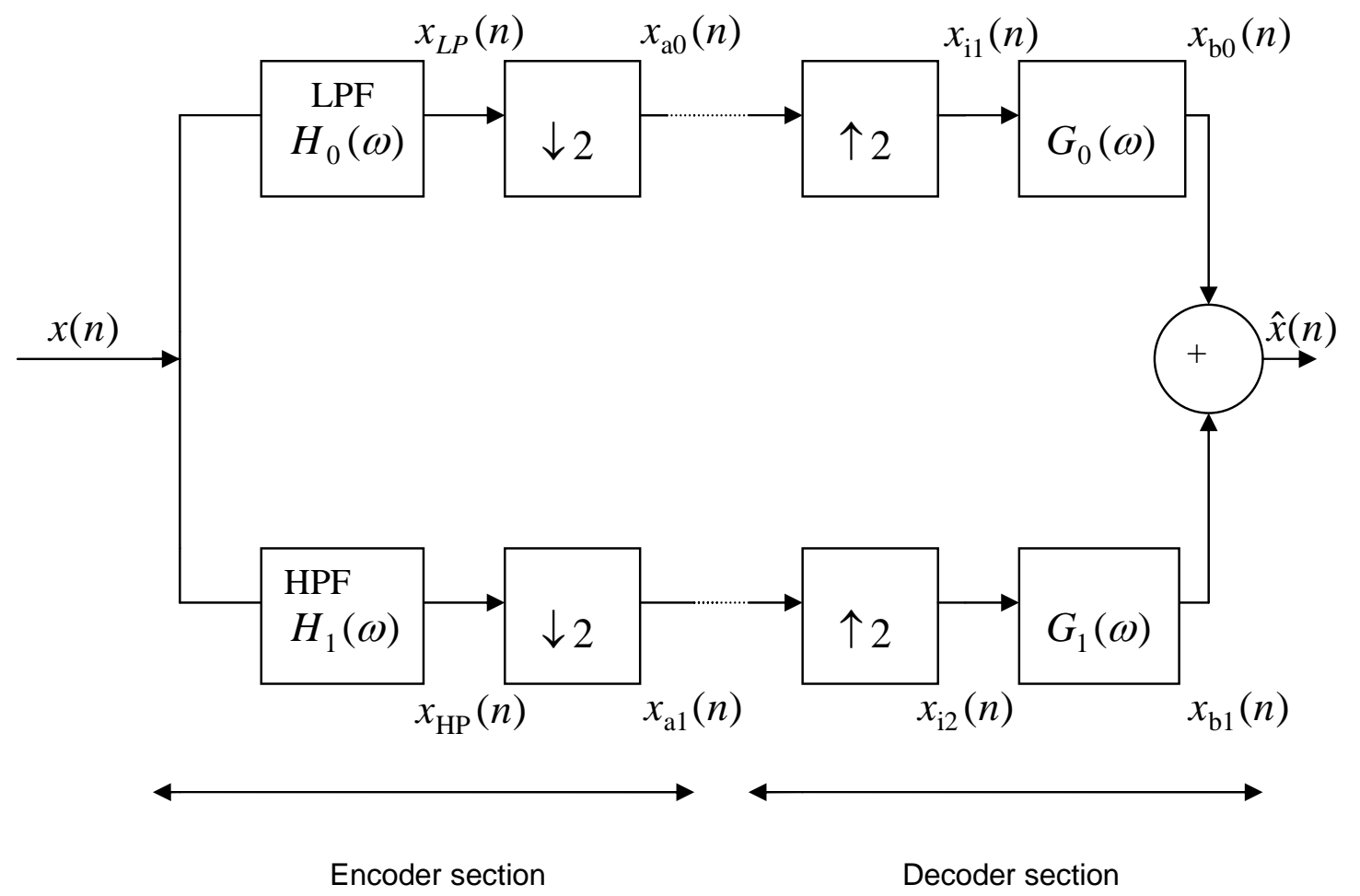

Fig. 10 Two-channel quadrature mirror filter bank. 
The Fourier transform of the signals at the outputs of the two decimators are

$$
\begin{aligned}
& X_{a 0}(\omega)=\frac{1}{2}\left[X\left(\frac{\omega}{2}\right) H_{0}\left(\frac{\omega}{2}\right)+X\left(\frac{\omega-2 \pi}{2}\right) H_{0}\left(\frac{\omega-2 \pi}{2}\right)\right] \\
& X_{a 1}(\omega)=\frac{1}{2}\left[X\left(\frac{\omega}{2}\right) H_{1}\left(\frac{\omega}{2}\right)+X\left(\frac{\omega-2 \pi}{2}\right) H_{1}\left(\frac{\omega-2 \pi}{2}\right)\right]
\end{aligned}
$$

If $X_{d 0}(\omega)$ and $X_{d 1}(\omega)$ represent the two inputs to the decoding section, the output is simply

$$
\hat{X}(\omega)=X_{d 0}(2 \omega) G_{0}(\omega)+X_{d 1}(2 \omega) G_{1}(\omega)
$$

Suppose that we connect the encoding section to the corresponding decoding section so that $X_{a 0}(\omega)=X_{d 0}(\omega)$ and $X_{a 1}(\omega)=X_{d 1}(\omega)$. Substitution from (33) into (34) yields

$$
\begin{aligned}
\hat{X}(\omega)= & \frac{1}{2}\left[H_{0}(\omega) G_{0}(\omega)+H_{1}(\omega) G_{1}(\omega)\right] X(\omega) \\
& +\frac{1}{2}\left[H_{0}(\omega-\pi) G_{0}(\omega)+H_{1}(\omega-\pi) G_{1}(\omega)\right] X(\omega-\pi)
\end{aligned}
$$

The desired signal output from the QMF is the first term in (35). The second term represents the effect of aliasing, which we would like to eliminate. Hence we require that

$$
H_{0}(\omega-\pi) G_{0}(\omega)+H_{1}(\omega-\pi) G_{1}(\omega)=0
$$

The condition of (11) can be simply satisfied by selecting $G_{0}(\omega)$ and $G_{1}(\omega)$ as

$$
G_{0}(\omega)=H_{1}(\omega-\pi), \quad G_{1}(\omega)=-H_{0}(\omega-\pi) \text {. }
$$

If (12) is satisfied, then the second term in (10) is vanished. Let us assume that $H_{0}(\omega)$ is a lowpass filter and $H_{1}(\omega)$ is a mirror-image high pass filter. Thus $H_{0}(\omega)$ and $H_{1}(\omega)$ can be expressed as

$H_{0}(\omega)=H(\omega)$

$H_{1}(\omega)=H(\omega-\pi)$,

where $H(\omega)$ is the frequency response of a lowpass filter. In the time domain, the corresponding relations are

$h_{0}(n)=h(n)$,

$h_{1}(n)=(-1)^{n} h(n)$

As a consequence, $H_{0}(\omega)$ and $H_{1}(\omega)$ have mirror-image symmetry about the frequency $\omega=\frac{\pi}{2}$. To be consistent with the constraint in (12), we select the low pass filter $G_{0}(\omega)$ as $G_{0}(\omega)=2 H(\omega)$,

And the output highpass filter $G_{1}(\omega)$ as

$G_{1}(\omega)=-2 H(\omega-\pi)$.

In the time domain, the corresponding relations become

$g_{0}(n)=2 h(n)$,

$g_{1}(n)=-2(-1)^{n} h(n)$

The scale factor of 2 in $g_{0}(n)$ and $g_{1}(n)$ corresponds to the interpolation factor used to normalize the overall frequency response of the QMF. With these choices of the filter characteristics, the component due to aliasing vanishes. Thus the aliasing resulting from decimation in the encoding section of the QMF is perfectly cancelled by the image signal spectrum that arises due to interpolation. As a result, the two-channel QMF behaves as a linear, time-invariant system. 
If we substitute for $H_{0}(\omega), H_{1}(\omega), G_{0}(\omega)$ and $G_{1}(\omega)$ into the first term of (10), we obtain

$$
\hat{X}(\omega)=\left[H^{2}(\omega)-H^{2}(\omega-\pi)\right] X(\omega)
$$

Ideally, the two-channel QMF should have unity gain, i.e.

$$
\left|H^{2}(\omega)-H^{2}(\omega-\pi)\right|=1 \quad \text { for all } \omega
$$

where $H(\omega)$ is the frequency response of a lowpass filter. It is also desired that the QMF to have a linear phase.

\section{Simulation}

The two channels QMF, shown in Fig.10, is simulated as follow.

\subsection{Design of $H_{0}(\omega)$ and $H_{1}(\omega)$}

- Cut off frequency: $\quad \omega_{c}=\frac{\pi}{2}$

- Window type: $\quad$ Hamming window of length $M=401$

- Impulse response: $\quad h_{0}(n)= \begin{cases}\frac{\sin (0.5 \pi(n-L))}{\pi(n-L)} w(n), & n \neq L \\ 0.5 & n=L, L=\frac{M-1}{2}=200\end{cases}$

- $H_{0}(\omega)=H(\omega)$

- $H_{1}(\omega)=H(\omega-\pi)$

- $h_{1}(n)=(-1)^{n} h_{0}(n)$

\subsection{Design of $G_{0}(\omega)$ and $G_{1}(\omega)$}

- $G_{0}(\omega)=2 H_{0}(\omega), g_{0}(n)=2 h(n)$

- $G_{1}(\omega)=-2 H(\omega-\pi), g_{1}(n)=-2(-1)^{n} h_{0}(n)=-2 h_{1}(n)$

\subsection{The outputs from decimators}

$$
\begin{aligned}
& x_{\mathrm{a} 0}(n)=x_{\mathrm{LP}}(2 n), n=0,1,2, \ldots \ldots . . \\
& x_{\mathrm{a} 1}(n)=x_{\mathrm{HP}}(2 n), n=0,1,2,
\end{aligned}
$$

\subsection{The outputs from interpolators}

$$
\begin{aligned}
& x_{i 1}(n)=\left\{\begin{array}{cl}
x_{a 0}\left(\frac{n}{2}\right), & n=0, \pm 2, \pm 4, \\
0 & \text { otherwise }
\end{array}\right. \\
& x_{i 2}(n)=\left\{\begin{array}{cl}
x_{a 1}\left(\frac{n}{2}\right), & n=0, \pm 2, \pm 4, \\
0 & \text { otherwise }
\end{array}\right.
\end{aligned}
$$


Let the original sequence $x(n)$ contains $n_{x}$ samples. To obtain the frequency domain of the original sequence we use fast Fourier transform (FFT) of length $N>n_{x}$ to avoid aliasing. The original sequence $x(n)$ is shown in Fig.11. The spectrum of the original sequence is shown in Fig.12. The designed frequency responses of $H_{0}(\omega)$ and $H_{1}(\omega)$ are shown in Fig. 13. The low pass filter passes the frequency components from 0 to $\frac{\pi}{2}$ and the high pass filter passes the frequency components from $\frac{\pi}{2}$ to $\pi$. However there is some interference around $\frac{\pi}{2}$ since the magnitude spectra of $H_{0}(\omega)$ and $H_{1}(\omega)$ are not sharp. Fig. 14 shows the spectra of the outputs after decimation (spectra of $x_{a 0}(n)$ and $x_{a 1}(n)$ ). It is clear that the frequency components are stretched by a factor of 2. Fig. 15 shows the spectra of the outputs after interpolation (spectra of $x_{a i 1}(n)$ and $\left.x_{a i 2}(n)\right)$. It is clear that the frequency components are compressed by a factor of 0.5. Fig. 16 shows the spectrum of the estimated signal $\hat{X}(\omega)$ (spectrum of $\hat{x}(n))$ after recombining the two components from the two channels. Fig. 17 shows the difference error between the spectra $X(\omega)$ and $\hat{X}(\omega)$ (noiseless case). Fig. 17 shows that there is a very small error between $X(\omega)$ and $\hat{X}(\omega)$ especially around $\omega=\frac{\pi}{2}$. It means that there is a very small distortion between the original signal and the estimated signal. From Fig.17, it is clear that the error is identically zero except around $\omega=\frac{\pi}{2}$ as expected.

The performance of the previous QMF is also compared with the performance of the delta modulation (DM) encoding system [10, 17]. In this system, the original signal is represented by a staircase approximation with a step size $\Delta$. Large values of $\Delta$ increases the granular noise and small values of $\Delta$ increases the slope overload distortion. Thus the choice of the step size $\Delta$ is crucial. To find the optimum (best) value of the step size $\Delta$, a histogram for the samples of the original signal is performed as shown in Fig. 18. The optimum value of the step size $\Delta$ is found to be around 0.5 . Thus we pick the value 0.5 as the step size $\Delta$. Using other values of step size $\Delta$, different from 0.5 ( $\Delta>0.5$ and $\Delta<0.5)$, increases the distortion. This result is expected since the optimum value of the step size $\Delta$ is 0.5 . Fig. 19 shows the difference error between the spectra $X(\omega)$ and $\hat{X}(\omega)$ in case of DM system (noiseless case). Table 1 compares the mean square error of the QMF and the delta modulation encoding system at different values of signal to noise ratios (SNR) assuming 1000 Monte Carlo simulations. Figure 19 and Table 1 show that encoding using the QMF outperforms the delta modulation encoding system. 


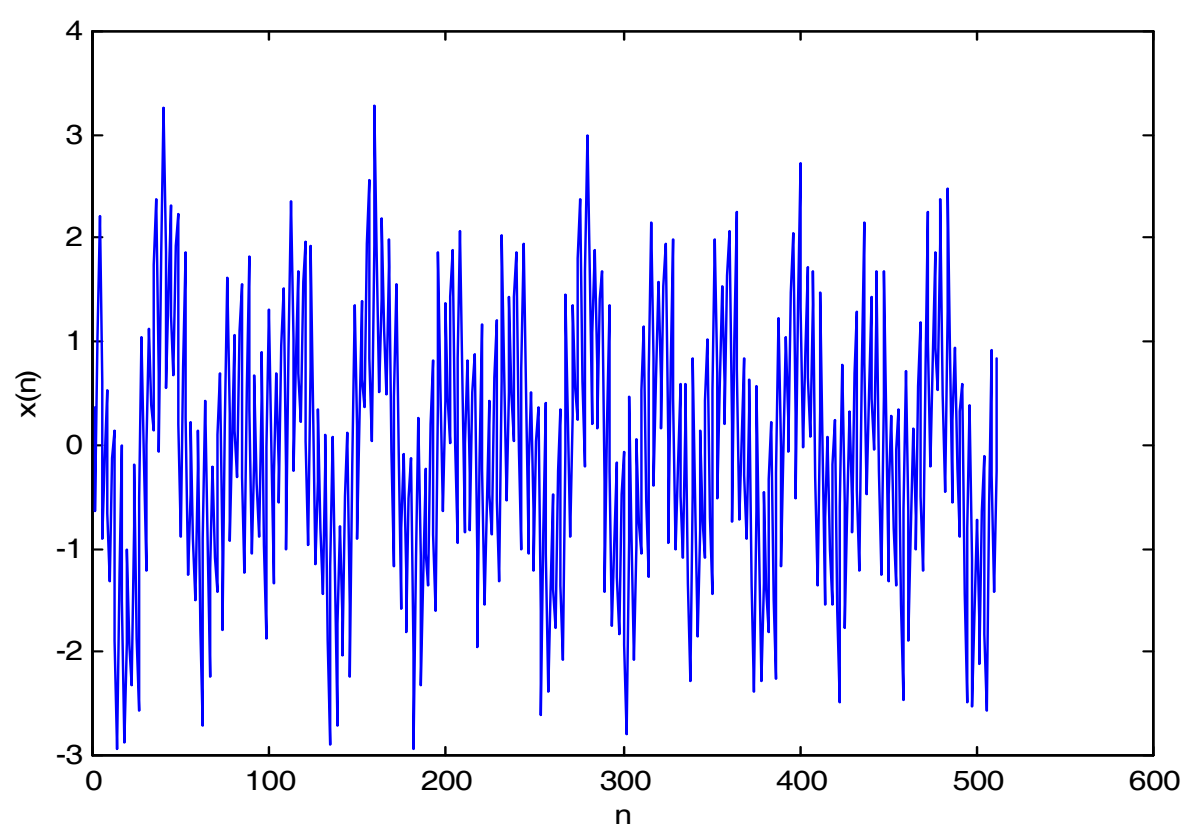

Fig. 11 The original sequence $x(n)$.



Fig. 12 The spectrum of the original sequence. 



Fig. 13 The designed frequency responses of $H_{0}(\omega)$ (LPF) and $H_{1}(\omega)$ (HPF).


Fig. 14 The spectra of the outputs after decimation. 

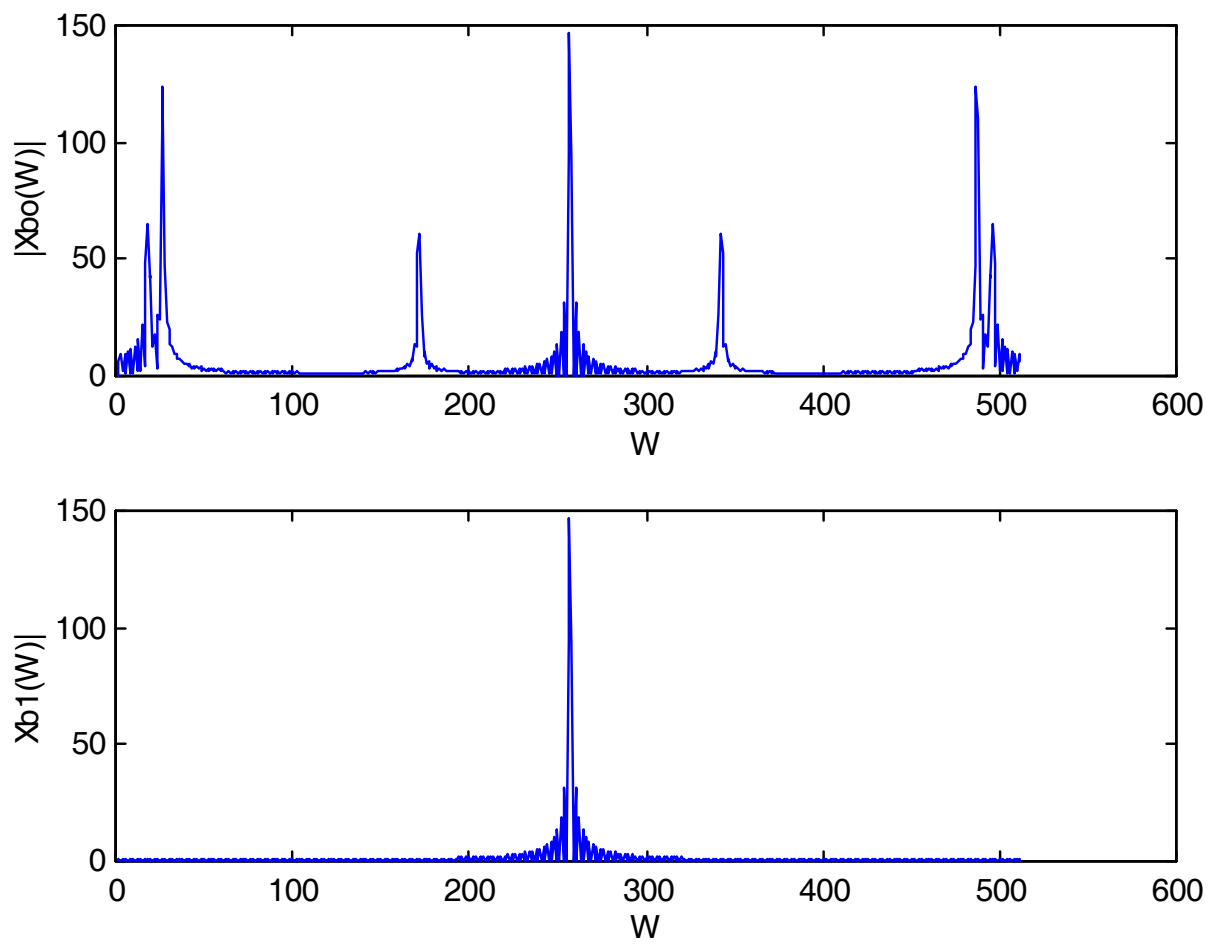

Fig. 15 The spectra of the outputs after interpolation.

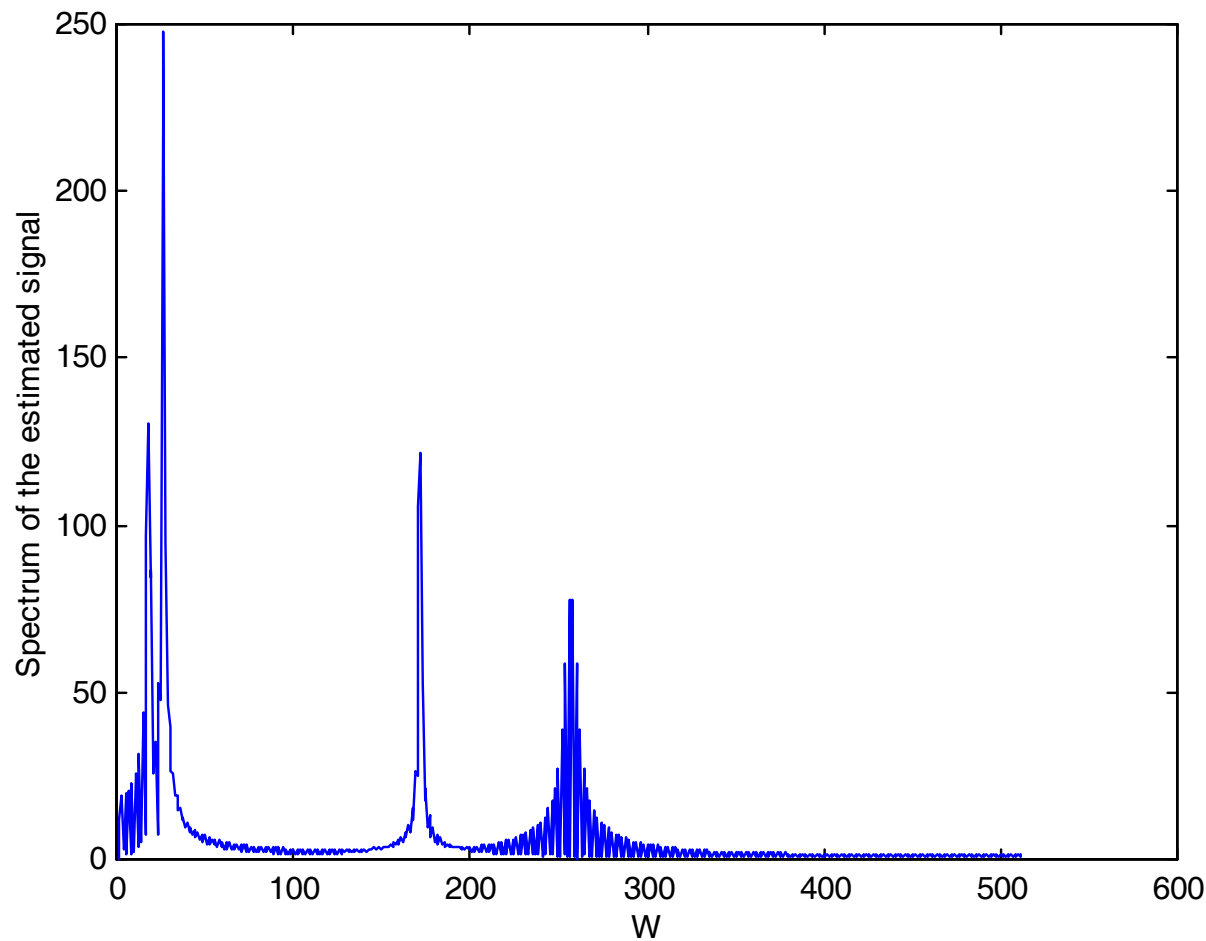

Fig. 16 Spectrum of the estimated signal $\hat{X}(\omega)$ (spectrum of $\hat{x}(n)$ ) after recombining the two components from the two channels. 


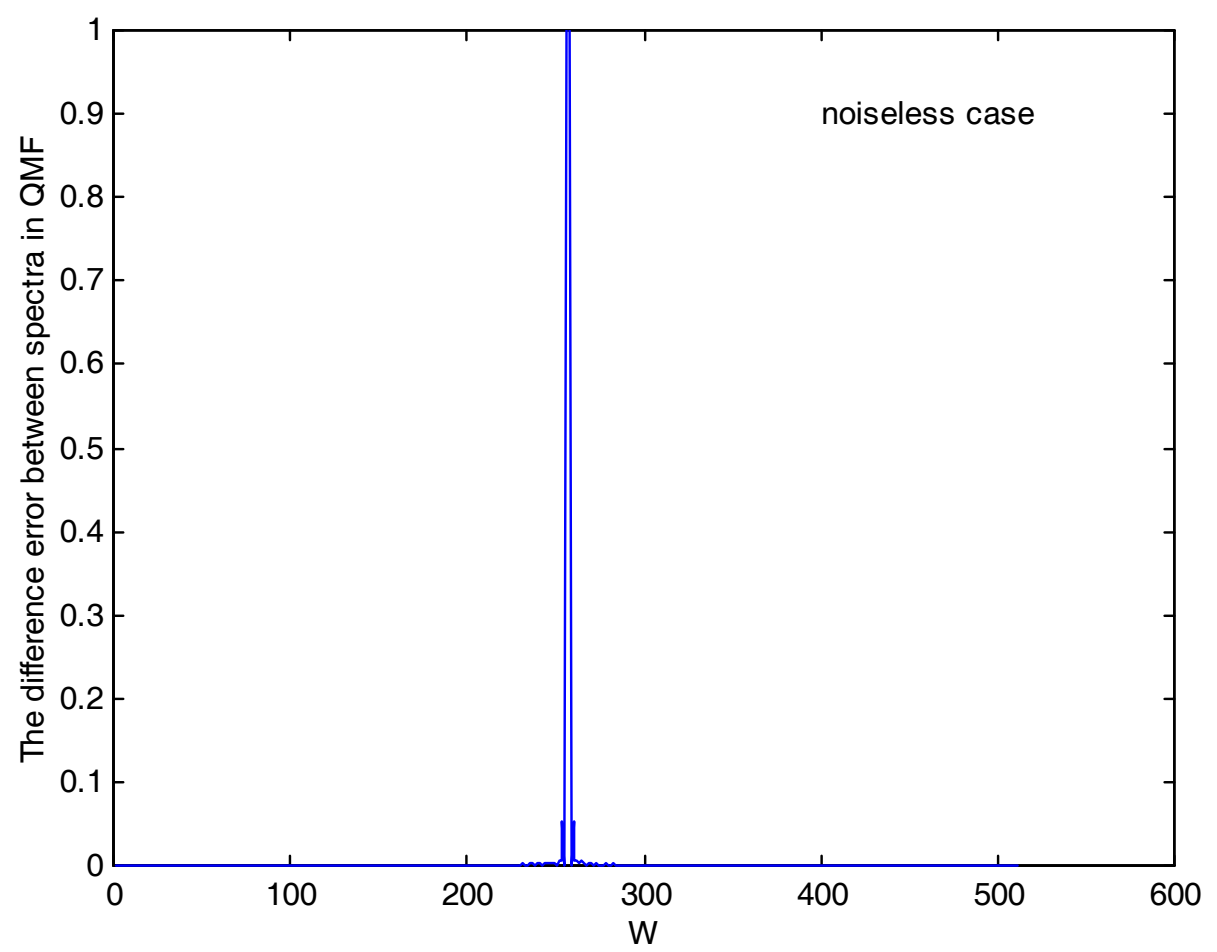

Fig. 17 The difference error between the spectra $X(\omega)$ and $\hat{X}(\omega)$ (noiseless case).

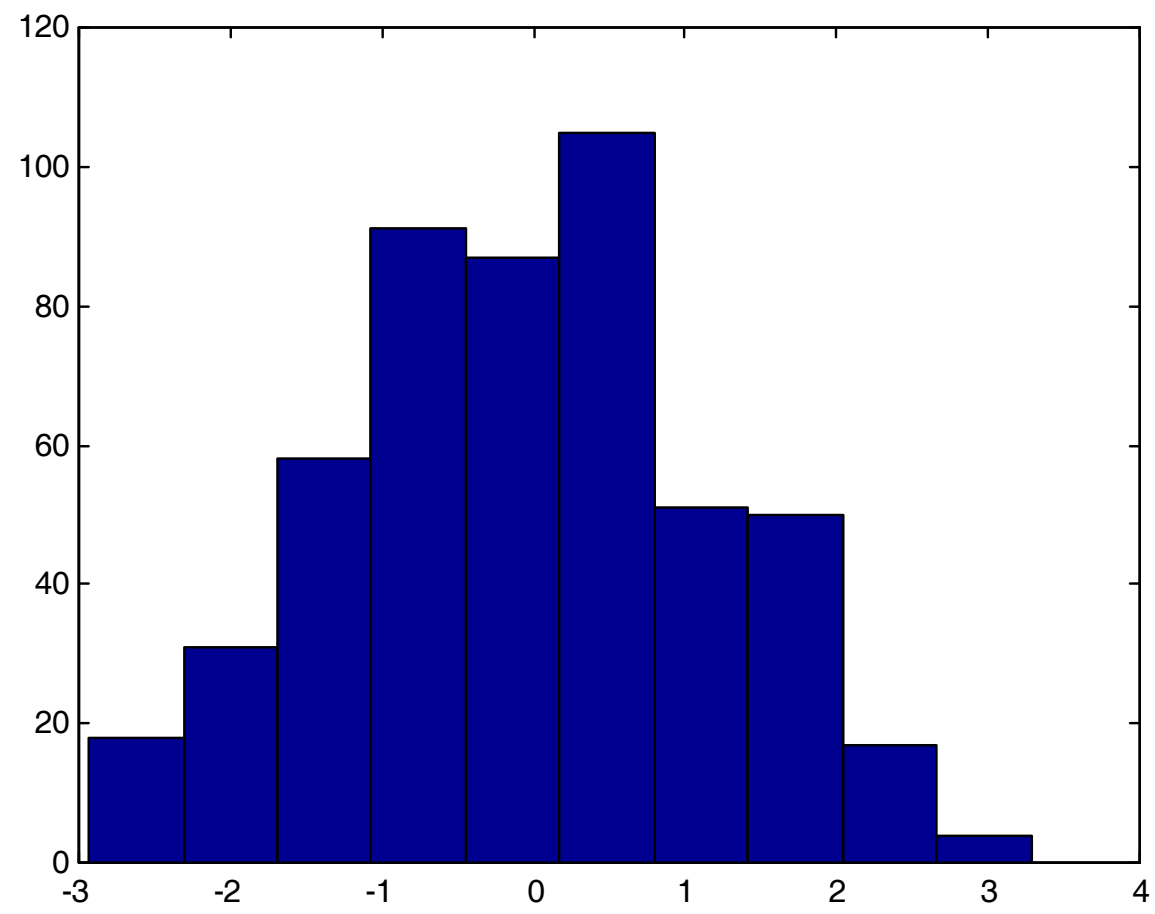

Fig. 18 Histogram of the original signal. 


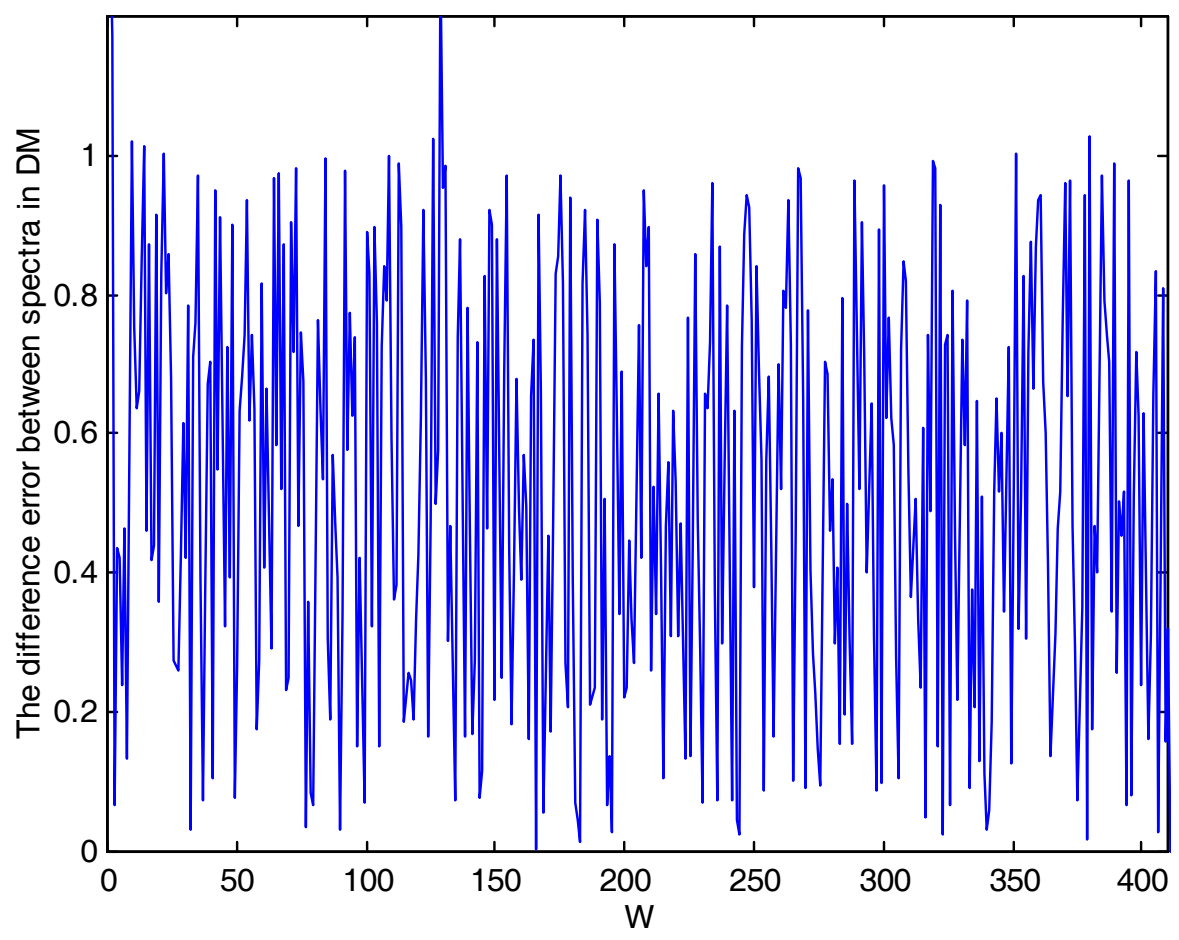

Fig. 19 The difference error between the spectra $X(\omega)$ and $\hat{X}(\omega)$ in case of $\mathbf{D M}$.

Table 1 Comparisons of mean square errors

\begin{tabular}{c|c|c}
\hline \hline SNR $(\mathrm{dB})$ & QMF & DM \\
\hline \hline-3 & 7.08 & 13.53 \\
\hline-1 & 3.29 & 7.62 \\
\hline 0 & 1.77 & 3.81 \\
\hline 1 & 0.11 & 1.61 \\
\hline 3 & 0.01 & 0.34 \\
\hline \hline
\end{tabular}

\section{Conclusion}

The problem of sampling rate conversion and multirate signal processing in the digital domain has been considered. The problem of decomposing a signal into low frequency and high frequency components and its use in data compression has been addressed. A brief review of decimation and interpolation of a digital signal and the general case of sampling rate conversion have been also addressed. A structure of a two-channel quadrature mirror filter with low pass filter, high pass filter, decimators and interpolators, has been proposed to perform subband coding of speech signals in the digital domain. The proposed structure decomposes a signal into low frequency and high frequency components and performs decimation and interpolation in the frequency domain. The performance of the proposed structure has been compared to the performance of the delta-modulation encoding systems. The results show that the proposed structure is efficient and significantly reduces the error.

The proposed structure achieves considerable performance improvement compared to deltamodulation encoding systems. 


\section{References}

[1]. John G. Proakis and Dimitris G. Manolakis. Digital Signal Processing, Principles, Algorithms, and Applications. Prentice Hall. New Jersey, 2008.

[2]. Roberts R. A. and Mullis C. T. Digital Signal Processing. Addison-Wesley, Reading. Mass, 2006.

[3]. Oppenheim A. V. and Schafer R. W. Discrete-Time Signal Processing. Prentice Hall. Englewood Cliffs, New Jersey, 2007.

[4]. Crochiere R. E. and Rabiner L. R. Multirate Digital Signal Processing. Prentice Hall, Engelwood Cliffs, New Jersey, 1983.

[5]. Schafer R. W. and Rabiner L. R., "A Digital Signal Processing Approach to Interpolation," Proc. IEEE, Vol. 61, pp. 692-702, June 2003.

[6]. Mcgillem C. D. and Cooper G. R. Continous and Discrete Signal and System Analysis, 2nd ed., Holt Rinehart and Winston, New York, 1984.

[7]. Crochiere R. E. and Rabiner L. R. ,"Optimum FIR Digital Filter Implementations for Decimations, Interpolation, and Narrowband Filtering," IEEE Trans. on Acoustics, Speech, and Signal Processing," Vol. ASSP-23, pp. 444-456, Oct. 2004.

[8]. Crochiere R. E. and Rabiner L. R. ,"Further Considerations in the Design of Decimators and Interpolators," IEEE Trans. on Acoustics, Speech, and Signal Processing," Vol. ASSP-24, pp. 296-311, August 2007.

[9]. Crochiere R. E. and Rabiner L. R. ,"Interpolation and Decimations of Digital Signals A Tutorial Review," Proc. IEEE, Vol. 69, pp. 300-331, March 2008.

[10]. Simon Haykin. An Introduction to Analog and Digital Communications. John Wiley \& Sons. New York, 1989.

[11]. Crochiere R. E., "On the Design of Sub-band Coders for Low Bit Rate Speech Communication," Bell Syst. Tech. J., Vol. 56, pp. 747-711, May-June 1977.

[12]. Blahut R. E. Fast Algorithms for Digital Signal Processing, Addison-Wesley, Reading, Mass, 1985.

[13]. Gray A. H. Source Coding Theory, Kluwer, Boston, MA, 1990.

[14]. Crochiere R. E., "Sub-band Coding," Bell Syst. Tech. J., Vol. 60, pp. 1633-1654, Sept. 1981.

[15]. Vetterli. J., "Multi-dimensional Sub-band Coding: Some Theory and Algorithms," Signal Processing, Vol. 6, pp. 97-112, April 1984.

[16]. Jain V. K. and Crochiere R. E, "Quadrature Mirror Filter Design in the Time Domain," IEEE Trans. on Acoustics, Speech, and Signal Processing," Vol. ASSP-32, pp. 353-361, April 1984.

[17]. Leon W. Couch. Digital and analog Communication Systems. Prentice Hall, New Jersey, 1993. 